\title{
Morphology evolution of thermally annealed polycrystalline thin films
}

\author{
A. González-González, ${ }^{1, *}$ G. M. Alonzo-Medina, ${ }^{2}$ A. I. Oliva, ${ }^{2}$ C. Polop, ${ }^{3}$ J. L. Sacedón, ${ }^{1}$ and E. Vasco ${ }^{1}$ \\ ${ }^{1}$ Instituto de Ciencia de Materiales de Madrid, Consejo Superior de Investigaciones Científicas (CSIC), 28049 Madrid, Spain \\ ${ }^{2}$ CINVESTAV-IPN, Unidad Mérida, Dept. Física Aplicada, AP 73-Cordemex, 97310 Mérida Yucatán, México \\ ${ }^{3}$ Dept. Física de la Materia Condensada, Universidad Autónoma de Madrid, 28049 Madrid, Spain \\ (Received 3 June 2011; revised manuscript received 28 July 2011; published 31 October 2011)
}

\begin{abstract}
Investigation of the morphology evolution of annealed polycrystalline $\mathrm{Au}(111)$ films by atomic force microscopy and x-ray diffraction leads to a continuous model that correlates such an evolution to local interactions between grains triggering different mechanisms of stress accommodation (grain zipping and shear strain) and relaxation (gap filling and grain rotation). The model takes into consideration findings concerning the in-plane reorientation of the grains during the coalescence to provide a comprehensive picture of the grain-size dependence of the interactions (underlying the origin of the growth stress in polycrystalline systems); and in particular it sheds light on the postcoalescence compressive stress as a consequence of the kinetic limitations for the reorientation of larger surface structures.
\end{abstract}

DOI: 10.1103/PhysRevB.84.155450

PACS number(s): 68.55.J-, 68.60.Bs, 81.15.Aa

\section{INTRODUCTION}

During the deposition of polycrystalline metal thin films grown according to the Volmer-Weber mode (VW), the evolution of the growth stress (which has its origin in the interaction between grains) exhibits several transitions (compressive $\rightarrow$ tensile $\rightarrow$ compressive) as the film thickness increases ${ }^{1}$ or the grain size raises for postdeposition annealing. ${ }^{1,2}$ These transitions have been correlated with the inherent stages to VW-type growth: (i) Nucleation and grain growth, (ii) grain coalescence and percolation, and (iii) closure and film growth. It is widely admitted that during stages (i) and (ii) the surface tension and the cohesive forces between close grains generate compressive and tensile stresses, respectively, with significant changes in the film morphology. ${ }^{3,4}$ However, the repulsive interactions that generate the compressive growth stress observed upon the film closure (postcoalescence stress hereafter) is less understood and currently a matter of intense debate ${ }^{5,6}$ since it appears in the thickness range of interest for nanotechnology. Several models on postcoalescence stress have been proposed so far in order to elucidate the origin of the repulsion between grains at this stage. An early model postulated that both compressive stresses generated during stages (i) and (iii) have the same origin: the Laplace pressure caused by the surface tension, which is counterbalanced in (ii) by the cohesive forces between grains. ${ }^{5,7}$ Later, Chason et al . proposed that the compressive stress in stage (iii) results from surface downhill currents, leading an excess of adatoms into the grain boundaries (GBs) to be inserted. ${ }^{8}$ A third model points out the elastic deformation field that experiences a surface on which a density of adatom has been deposited as the cause of the postcoalescence compressive stress. ${ }^{9}$

In this paper, we report experimental data concerning the morphology evolution of polycrystalline $\mathrm{Au}(111)$ films during thermal annealing. In particular, we present findings indicating that the coalescence involves the in-plane reorientation of the surface grains and the formation of pseudocoherent (i.e. low-angle and coincidence-site-lattice) GBs between them. Based on these results, we propose a model to address the morphology evolutions in terms of the rising of attractive/repulsive interactions between surface grains. Such a model offers a comprehensive picture of the phenomenon, which allows us to throw light on the origin actually in question ${ }^{1-9}$ of the postcoalescence compressive stress.

\section{EXPERIMENT}

Au films were deposited on $\mathrm{SiO}_{x}$-covered $\mathrm{Si}(111)$ substrates at a nominal temperature of $T_{\text {growth }}=100^{\circ} \mathrm{C}(0.28$ $T_{\text {melting }}$ ) and a pressure of $10^{-7}$ mbar. The film thickness was $d=200 \mathrm{~nm}$ for all samples with a deposition rate of $F=$ $10 \AA / \mathrm{s}$. This rate is two orders of magnitude higher than the typical rates $(\sim 0.1 \AA / \mathrm{s}),{ }^{10}$ which gives rise to a film microstructure ruled by kinetic limitations that hinder both the structural and morphological relaxations during deposition. ${ }^{11}$ Once deposited, the flux was stopped, and the samples were held at the growth temperature for an in-situ annealing (which enables the film relaxation) during $t_{a}=1.2 \times 10^{2}-1.2 \times 10^{5} \mathrm{~s}$ under an $\operatorname{Ar}$ flux $\left(P_{\mathrm{Ar}}=1 \mathrm{~atm}\right)$. Afterwards, the samples were cooled quickly down to room temperature and investigated by atomic force microscopy (AFM) using ultrasharp silicon tips (with a nominal radius of $2 \mathrm{~nm}$ and estimated aspect ratio on calibration samples of $2 m_{\text {tip }} \approx 1.5$ ) and by x-ray diffraction (XRD) in $\theta-2 \theta$ and $\phi$-scan geometries measured in an X'Pert four-circle diffractometer using $\mathrm{Cu} \mathrm{K}_{\alpha}$ radiation. The thus-prepared films exhibited an out-of-plane $\mathrm{Au}[111]$ texture.

\section{RESULTS}

Figures 1(a)-1(d) show the morphology evolution of the Au films with $t_{a}$, whereas Figs. $1(\mathrm{e})-1(\mathrm{~g})$ plot the variation of surface statistical parameters: roughness $\omega$ [Fig. 1(e), the root mean square (RMS) fluctuation of the surface heights], the in-plane size $\lambda$ of the surface features [Fig. 1(f), left-side axis; the mean diameter measured along the film plane of surface features, such as grains, grain bundles, or multigrain structures], out-of-film plane grain size $\lambda_{\perp}$ [Fig. 1(f), right-side axis; a measure of the crystal coherence along the film thickness determined by $\theta-2 \theta$ XRD using the Scherrer's equation], and the slope $\hat{m}$ at the bottom flanks of surface features [border slope hereafter, Fig. 1(g)]. Due to the heterogeneity of the surface features, the morphology analysis was performed at 


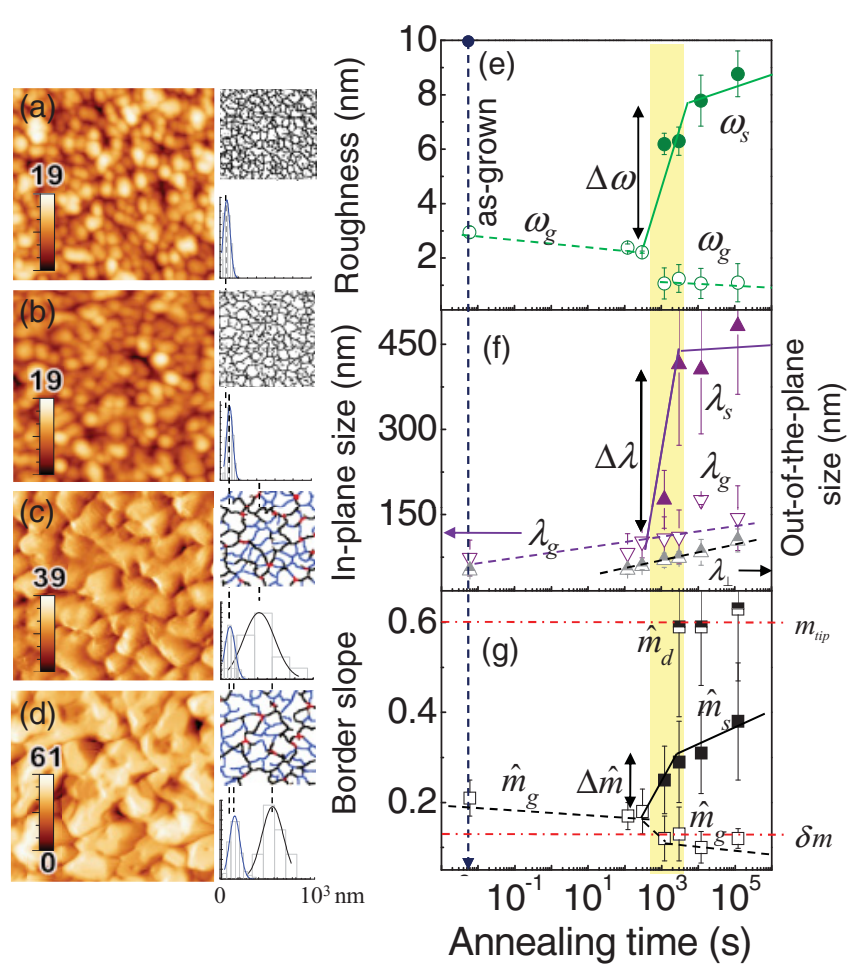

FIG. 1. (Color online) $1 \times 1 \mu \mathrm{m}^{2}$-sized AFM images of $200 \mathrm{~nm}$ thick polycrystalline $\mathrm{Au}$ films with annealing times of (a) $t_{a}=$ $0 \mathrm{~s}$ (as-grown), (b) $3 \times 10^{2} \mathrm{~s}$, (c) $3 \times 10^{3} \mathrm{~s}$, and (d) $1.2 \times$ $10^{4} \mathrm{~s}$. Upper insets: tessellated surface in which the white, black (blue) and red regions/curves depict the inner areas of the surface features, the external (internal) GBs, and surface discontinuities, respectively. Lower insets: size distributions of the different types of coexisting surface features. (e)-(g) Evolution of statistical morphology parameters at different-length scales (as described in the text): (e) roughness, (f) in-plane and out-of-plane sizes $\left(\lambda\right.$, left and $\lambda_{\perp}$, right axis, respectively) of the surface features, and $(\mathrm{g})$ border slope $\hat{m}$.

different length scales (in connection with characteristic sizes of each). Thus, the insets in Figs. 1(a)-1(d) correspond to: (upper) contour maps or tessellations of the AFM images in which the white, black, blue, and red regions/curves depict the areas of surface features, the GBs delimiting them (external GBs), the internal GBs delimiting the grains inside larger features identified as grain bundles or structures, and the surface discontinuities (grooves and holes), respectively; (lower) the size distributions of the different surface features coexisting in each sample. Consequently, the statistic parameters [as depicted in Figs. 1(e)-1(g)] for dissimilar length scales are defined using a set of indices referred to: (no index) generic information obtained on the AFM images before being ascribed to a particular surface feature; (subindex $g$ ) local data measured on the grain-size scale [e.g. $\omega_{g}$ intragrain roughness, $\lambda_{g}$ grain size, and $\hat{m}_{g}$ slope at the grain borders in Figs. 1(e)1(g), respectively]; (subindex $s$ ) analogous information but referred to multigrain structures $\left[\omega_{s}, \lambda_{s}\right.$, and $\hat{m}_{s}$ in Figs. 1(e)$1(\mathrm{~g})$, respectively]; and (subindex $d$ ) data acquired on surface discontinuities $\left[\hat{m}_{d}\right.$ in Fig. $\left.1(\mathrm{~g})\right]$. Considering that the GBs between touching features emerge toward the surface at a gap region confined laterally by the feature borders, a threshold border slope of $\delta m=0.15$ was chosen (as discussed below) to distinguish between internal GBs (those with $\hat{m} \leqslant \delta m$ ) and external ones $\left(\delta m<\hat{m}<m_{\text {tip }}\right)$. Beyond, the surface discontinuities are characterized by $\hat{m} \geqslant m_{\text {tip }}$, where $2 m_{\text {tip }} \approx$ $1.2-1.3$ corresponds to the effective aspect ratio of the AFM tips measured on the surface of the roughest sample (i.e. $m_{\text {tip }}$ is the appraisable maximum slope). The morphology evolution of the annealed films is described below in terms of: (i) grain growth and coalescence through $t_{a}$ dependence of $\lambda$ and (ii) grain deformation via the $t_{a}$-dependences of $\hat{m}$ and $\omega$ since $\Delta \lambda$ ascribed to the grain deformation is much lower than the dispersion in $\lambda$ due to the grain growth.

The as-grown sample [Fig. 1(a), $t_{a}=0$ ] exhibits a surface morphology formed by an arrangement of rounded features with uniform shapes and unimodal size distribution. The fact that such features have no internal GBs and that their in-plane and out-of-plane sizes are in the same range $\lambda \approx \lambda_{\perp} \sim 50$ $70 \mathrm{~nm}$ allows us to identify them as surface equiaxed singlecrystal grains (i.e. $\lambda_{g} \equiv \lambda$ ). Surface grains with abnormal large sizes out of the size distribution are not detected. As a result of the thermal annealing, two morphology regimes separated by a narrow transition [yellow bar in Figs. 1(e)-1(g)] are distinguished. [Early regime, Fig. 1(b)] For short annealing times $\left(t_{a} \leqslant 3 \times 10^{2} \mathrm{~s}\right)$, the as-grown morphology is roughly preserved. In this regime, the grains grow ( $\lambda_{g}$ increases) and expand laterally such that the gaps between them become shallow (the gap depth, that is $\propto \omega$, decreases) and smoother ( $\hat{m}_{g}$ drops). [Transition, Fig. 1(c)] For intermediate annealing times $\left(3 \times 10^{2} \mathrm{~s}<t_{a}<1.2 \times 10^{4} \mathrm{~s}\right)$, the film morphology changes (a melted-like morphology emerges) as revealed by the large variations of the statistical parameters $\Delta \omega, \Delta \lambda$, and $\Delta \hat{m}$. The fact that $\lambda$ rises from $\lambda_{g}$ up to $4 \lambda_{g}$ suggests that this change is related to the peer-to-peer coalescence of the surface grains (understood here as a type of grain welding) into irregularly shaped structures (i.e. $\lambda_{s} \equiv \lambda$ ) rather than normal or abnormal grain growth phenomena. This suggestion is supported by the following evidence and/or arguments: (a) Sections of internal GBs, which would correspond to the external GBs of the departing grains before coalescing, can be distinguished on the structure surfaces [blue curves in the upper inset of Fig. 1(c)]. This evidence is corroborated by the fact that the size of the areas delimited by the internal GBs shows a dependence on $t_{a}$ similar to that found in the early regime for $\lambda_{g}\left(t_{a}\right)$, which implies that such areas correspond to the inner grain surfaces. (b) The different surface features, once classified in grains/structures (features without/with internal GBs), exhibit unimodal size distributions around $\lambda_{g}$ and $\lambda_{s}$, respectively. (c) The irregular shapes of the large structures [as shown by the contour maps; compare upper insets in Figs. 1(a) and 1(d)] suggest that the growth mechanisms by atomic exchange between grains that compete with each other in which the shapes of the surviving grains are preserved (e.g. Ostwald-ripening ${ }^{12}$ ) play a minor role in the formation of such structures. [Late regime, Fig. 1(d)] Once the grain coalescence is completed (for $t_{a}>1.2 \times 10^{4} \mathrm{~s}$ ), the film surface is covered by large irregular structures, which are well separated from each other by external GBs and surface discontinuities. Internal GB sections are still visible on the surfaces of such structures, revealing their granular origin. In this regime, the statistical parameters exhibit behaviors opposed at different 
length scales: whereas the large structures stop growing $\left(\lambda_{s}\right.$ remains constant) and shrink laterally generating deeper and sharper gaps (both $\omega_{s}$ and $\hat{m}_{s}$ increase), and uncovered regions of the film bulk occasionally; the inner grains slightly grow ( $\lambda_{g}$ continues rising), and the gaps between them tend to disappear ( $\hat{m}_{g}$ decreases) such that structure tops are smoothed $\left(\omega_{g}\right.$ remains low $)$.

On the other hand, the grain growth kinetics reveal that, whereas the shapes [in particular the $\lambda_{g} / \lambda_{\perp}$ ratio with $\lambda_{g} \propto$ $t_{a}{ }^{0.14 \pm 0.02}$ and $\lambda_{\perp} \propto t_{a}{ }^{0.10 \pm 0.01}$, as estimated from Fig. 1(f)] of the surface grains are roughly conserved $\left(\lambda_{g} / \lambda_{\perp}=\right.$ $1.7 \pm 0.3)$ during thermal annealing, the structures are formed preferentially along the film surface $\left(\lambda_{s} / \lambda_{\perp}=1.7 \rightarrow\right.$ $4.4)$, which indicates that the grain coalescence during the transition [Fig. 1(c)] involves mainly surface processes. This assertion is supported by the fact that the coarsening exponents $p=\log (\lambda) / \log \left(t_{a}\right)[p=0.14$ (in-plane) and $p=0.10$ (out-of-plane)] are lower than $p=1 / 4$ (exponent predicted for an ideal surface grain growth, ${ }^{13}$ see discussion below); and it is in accordance with the low annealing temperature $\left(T_{a}=100^{\circ} \mathrm{C}\right)$ since higher temperatures are required to activate bulk recrystallization processes. Indeed, a similar morphology evolution to that obtained here was found for thinner (30 nm-thick) polycrystalline $\mathrm{Au}$ films subjected to dynamic annealing using temperature ramps. ${ }^{14}$ Despite that the morphology of a $200 \mathrm{~nm}$-thick film grown by VW should correspond to a postclosure [stage (iii)] growth regime, the above evidence suggests that the surface grains keep their tendencies to coalesce and percolate, which are typical of a VW stage (ii). These delayed tendencies of the surface grains are addressed by the structure zone model [zones I and $\mathrm{T}$ (Ref. 15)] that predicts for our deposition conditions (namely, $T_{\text {growth }} \approx 0.28 T_{\text {melting }}$ and $F=10^{2} \times$ typical deposition rate), a film microstructure characterized by grains stacked in columns with the surface grains being belatedly generated by secondary nucleation phenomena. In short, we can conclude from our results that, with regard to the coalescence and grain growth induced by postdeposition annealing at moderate temperatures, the behavior of the layer of the surface grains is equivalent to that of a freestanding thinner film with thickness $d \approx \lambda_{\perp}$. In our study, the polycrystalline film bulk below the surface layer allows us to minimize any potential influences of the substrate on the morphology evolution of the annealed films.

The in-plane texture of the annealed films was investigated by means of both the azimuthal $\phi$ dependence of the distribution of surface slopes $m$ [stereographic plots in Fig. 2, $N(m$, $\phi)]$ and DRX $\phi$-scan (right-hand upper spectra in Fig. 2). These two techniques provide complementary information at different length scales (mesoscopic and macroscopic by $N(m, \phi)$ and $\phi$-scan, respectively) as follows: since the preferential out-of-plane $\mathrm{Au}[111]$ orientation of the grains, (a) $N(m, \phi)$-based analysis assumes that, for large enough single-crystal grains in which the surface tension does not control their shapes, the azimuthal distribution of their facets (a slope range can be ascribed to each facet) is connected with the in-plane orientation of the grains and thus with the in-plane texture of the film by making statistics over representative AFM images. On these premises, the $N(m, \phi)$ based analysis has been previously used to study the in-plane

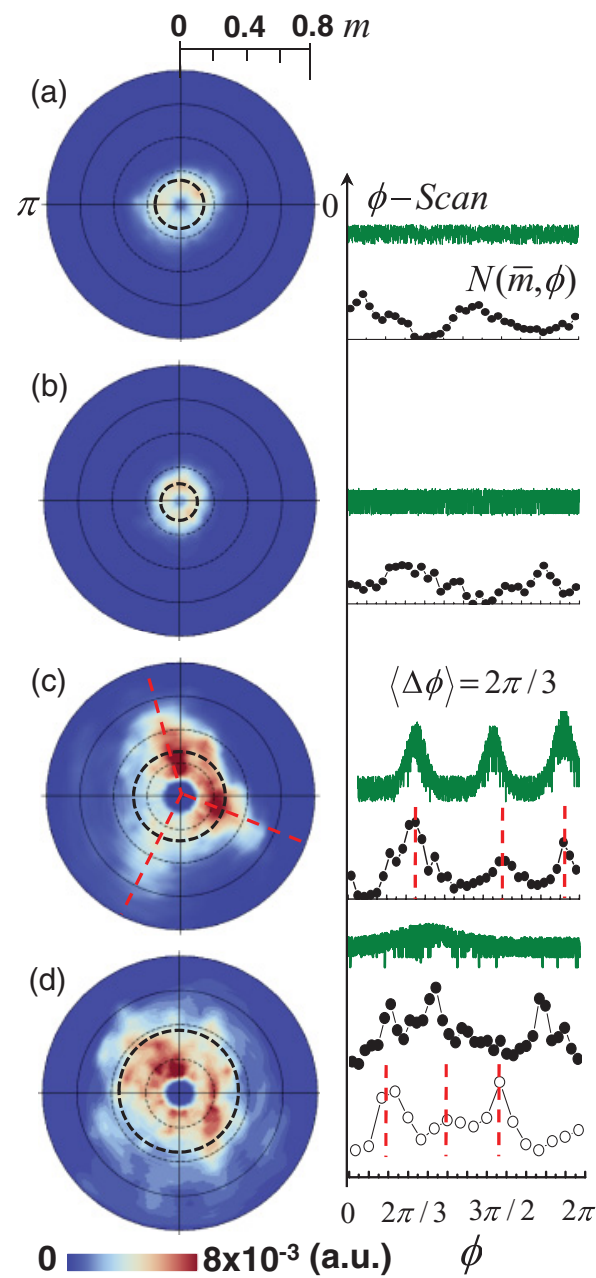

FIG. 2. (Color online) (Left-hand) Normalized $\phi$ dependence of the slope distribution $N(m, \phi)$ for 200-nm-thick polycrystalline $\mathrm{Au}(111)$ films annealed at different times: (a) $t_{a}=0 \mathrm{~s}$ (as-grown), (b) $3 \times 10^{2} \mathrm{~s}$, (c) $3 \times 10^{3} \mathrm{~s}$, and (d) $1.2 \times 10^{4} \mathrm{~s}$. (Right-hand) XRD $\phi$-scan spectra around $\mathrm{Au}(420)$ planes (upper spectrum), and $\phi$ profiles $N(\bar{m}, \phi)$ acquired at inter- $(\bullet)$ and intrastructure (०) scales.

texture (termed there as the surface orientation map, SOM) in nanostructured systems ${ }^{16,17}$ and polycrystalline films ${ }^{18}$ at space scales far below the DRX lateral resolution limit. Details concerning AFM image processing to compute $N(m, \phi)$ are reported elsewhere. ${ }^{19}$ On the other hand, (b) DRX $\phi$-scan analysis uses (in our case) the azimuthal distribution of the population of $18.4^{\circ}$-tilted $\mathrm{Au}(420)$ planes to investigate the film in-plane texture at x-ray spot-size scale. The azimuthal profiles $N(\bar{m}, \phi)$, where $\bar{m}$ corresponds to the mode value of each distribution, are included in Fig. 2 (right-hand lower plots) to compare with the corresponding DRX $\phi$-scan spectra.

Figure 2(a) discloses that, during the deposition, a random in-plane texture is generated (i.e. a ringlike structure emerges), which is preserved for $t_{a} \leqslant 3 \times 10^{2} \mathrm{~s}$ [early regime in Fig. 2(b)]. During the coalescence of the surface grains [transition in Fig. 2(c)], a threefold axis in-plain texture is suddenly formed as revealed by the profile $N(\bar{m}, \phi)$, where three broad bands spaced $\langle\Delta \phi\rangle=2 \pi / 3$ are distinguished. This result indicates plausibly that coalescence involves the 
rotation of the in-plane orientations of the neighboring grains (process termed reorientation hereafter) in order to minimize the misorientation between them and/or to decrease the GB contribution to the system energy. DRX $\phi$-scan shows that the formation of such an in-plane texture is a massive phenomenon that spreads up hundreds of microns. Once completed, the grain coalescence [late regime in Fig. 2(d)], the threefold axis in-plane texture is roughly preserved at nanometric scale inside single structures (lower profile). However, it worsens progressively at mesoscopic scale as several structures are considered (the bands in $N(\bar{m}, \phi)$ are not longer evenly spaced; intermediate profile) and disappearing at macroscopic scale (the $\phi$-scan spectrum shows a broad band; upper profile). The progressive disappearance of the in-plane texture as the inspected area is larger suggests the existence of different in-plane reorientation mechanisms operating to dissimilar scales (in particular, those responsible for the intra- and interstructure reorientation) and discards the possibility that the surface large structures [in Fig 1(d)] result from a grain growth based upon texture selection processes. ${ }^{20}$ The fact that the polycrystalline film bulk isolates mechanically the surface layer from the stiffener Si substrate (as discussed above) makes possible the reorientation giving rise to the in-plane texture, which would be frustrated otherwise given the higher shear modulus of $\mathrm{Si}$.

A detailed inspection of the DRX $\phi$-scan bands [in Fig. 2(c)] that correspond to the threefold axis in-plane texture is displayed in Fig. 3(a). The fact that the bands are wider (with full width at half maximum, FWHM $\sim \pi / 6$ ) than those of single-crystal $\mathrm{Au}(\mathrm{FWHM}<\pi / 45)$ reveals the poor crystalline quality of the in-plane texture. This result

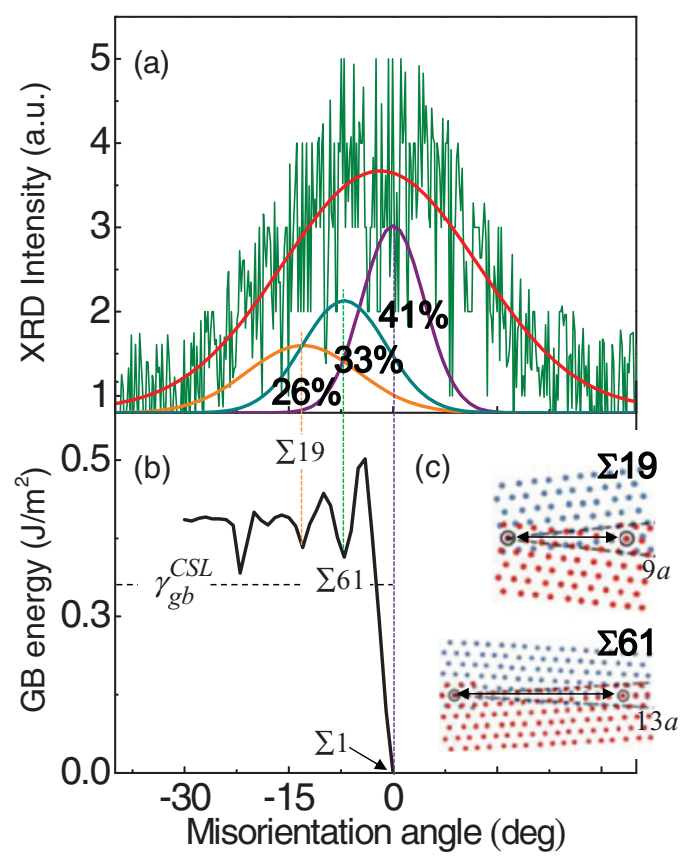

FIG. 3. (Color online) (a) Fit of a DRX $\phi$-scan band to pseudoVoigt peaks ascribed to the relative populations (labeled in \%) of three types of pseudocoherent GBs: $\Sigma 1\left(\theta=0^{\circ}\right), \Sigma 61\left(7^{\circ}\right)$ and $\Sigma 19\left(13^{\circ}\right)$; (b) $\theta$ dependence of $\gamma_{g b}$ computed for Au(111) using EAM (Ref. 23); and (c) atomic configurations of the CSL GBs whose fitting periodicities are indicated in atomic spacing, $a$. suggests that reorientation of the surface grains that underlies the in-plane texture involves the formation of pseudocoherent GBs (with no negligible misorientation angles $\theta \leqslant \frac{1}{2}$ FWHM) rather than a perfect fit between the coalescing grains. Different kinds of pseudocoherent GBs are possible: (a) low-angle GBs with $\theta=0-4^{\circ}$ (we have included here the coherent GBs or no GBs for $\theta=0$ ), and (b) pretty fitting kinds of GBs in coincidence-site-lattice (CSL) configuration. Whereas the former involves a complete grain reorientation to give rise to a quasiperfect fit (at nearest neighbor atoms) of the GBs, the latter implies partial reorientations towards GB configurations [with long-range fitting periodicities; sketches in Fig. 3(c)] that correspond to local minima of the dependence of GB energy $\left(\gamma_{g b}\right)$ on the misorientation angle $\theta$. For $\mathrm{Au}(111)$, details in Refs. 21 and 22, two types of CSL GB-configurations with $\theta \leqslant \pi / 12$ are available at $\theta=7^{\circ}(\Sigma 61)$ and $\theta=13^{\circ}(\Sigma 19)$. To estimate the contribution of each kind of GB to the in-plane texture, the DRX $\phi$-scan band was fitted to pseudo-Voigt peaks ascribed to three GB populations: low-angle GBs $(\Sigma 1), \Sigma 61$, and $\Sigma 19$, whose positions were fixed to $\theta=0^{0}, 7^{0}$, and $13^{0}$, respectively. The solid line in Fig. 3(a) represents the convolution of the peaks corresponding to the best fit, which was obtained for relative populations of each kind of GB of $\cong 41 \%(\Sigma 1), 33 \%(\Sigma 61)$ and $26 \%(\Sigma 19)$. The $\theta$ dependence of $\gamma_{g b}$ computed for Au(111) using the embedded-atom model (EAM) proposed by Mei et al. ${ }^{23}$ for fcc metals is plotted in Fig. 3(b). The thermodynamic magnitudes thus-obtained [viz, $\gamma_{s}$ surface energy and $\left.\gamma_{g b}(\theta)\right]$ by EAM are in good agreement with those reported by Wen and Zhang. ${ }^{24}$

\section{DISCUSSION}

In order to address the experimental evidence described above (recapitulating them: thermal annealing-induced morphology changes typify by single-crystal small grains that expand laterally, making shallow GBs up to coalesce into in-plane textured larger structures, which in turn shrink making deeper GBs) in terms of the interactions between grains and/or structures, we propose the following model considering the expansion and the shrinkage of the surface features as the results of the attraction and the repulsion between them, respectively:

\section{A. Model}

\section{System geometry}

The geometric and/or physical meanings of the main parameters involved in our model are illustrated in Fig. 4(a). We idealize the morphology of the polycrystalline thin film as formed by an array of round-shaped elastic grains (whose elastic constants are summarized in Ref. 25), which are separated from each other by a network of narrow gaps (with average gap width $\Delta \ll$ grain size $\lambda$ ). These grains interact to each other via: (i) long-range interactions (ruled by the surface tension) through the gap that extends a depth $h \propto \omega$ down to the grain junction ( $\Gamma$ point), where the neighboring grains come into contact with each other. (ii) Short-range interactions at the GBs (contact forces) that extend beyond the grain junction through the polycrystalline film bulk. This description allows us to identify the gap as the region in which the GBs emerge 


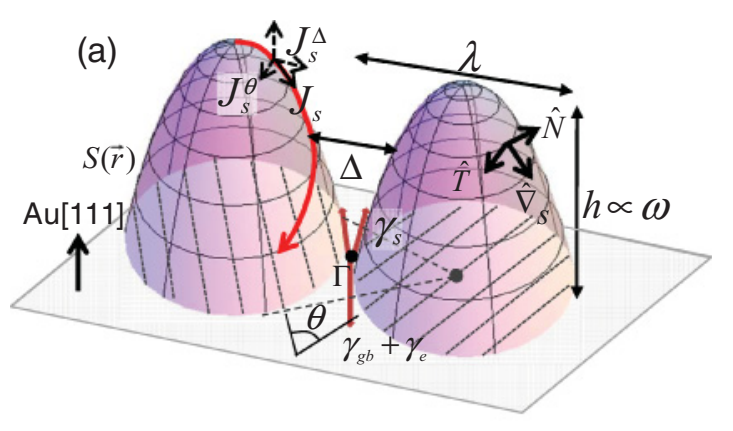

(b)

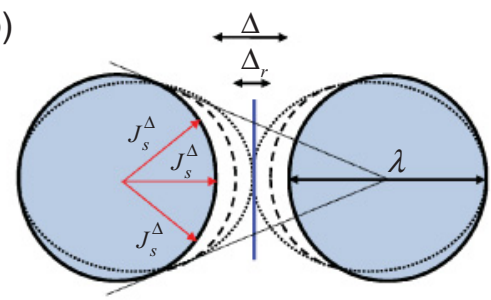

(c)

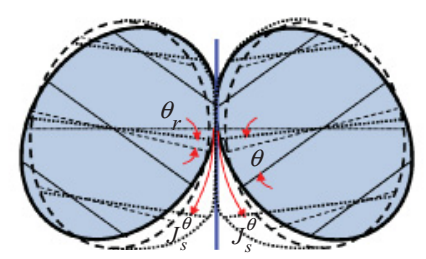

FIG. 4. (Color online) (a) Sketch of two interacting grains illustrating the main physical and geometrical parameters considered in the model. (b) and (c) Top view schematic representations of the mechanisms of relaxation by surface currents $\left(J_{s}^{\Delta}\right.$ and $\left.J_{s}^{\theta}\right)$ and accommodation by strain $\left(\Delta_{r}\right.$ and $\left.\theta_{r}\right)$ of the growth stress generated during the grain coalescence in both normal and azimuthal directions (respectively). Dashed (dotted) grain contour showing the morphology transformations of the grain shapes (the original ones being depicted by solid lines) as a result of the plastic (elastic) deformations.

toward the surface. The model assumes a cross-section of interaction between grains through the gap of $\alpha \lambda \omega / 2$, where $\alpha=\pi / 3$ denotes the in-plane contact angle for a compact array of grains. As sketched in Fig. 4(a), the interactions are described using a local system of three orthogonal unit vectors defined at each site of the film surface $S(\vec{r})$, where $\vec{r}$ denotes the position vector on the film plane; these are: $\hat{N}$, normal vector to the surface; $\hat{T}$, tangent vector to the level curves $S(\vec{r}) \equiv$ const.; and $\hat{\nabla}_{S}$, the surface gradient vector.

\section{Longitudinal interactions [Fig. 4(b)]}

The scenario of interactions between neighboring surface grains is the following one: in the early regime, attractive forces of traction between the opposite faces of the close grains arise as a consequence of the tendency of the system to drop its surface energy. These forces generate a normal tensile stress $\sigma_{N} \propto-\left(\hat{\nabla}_{s} 2 \gamma_{s}\right) \hat{N}>0$ [where $2 \gamma_{s}$ denotes the surface energy saved as a result of the perfect coalescence between two grains] on the grain faces within the gap region. Thus-generated $\sigma_{N}$ produces a phenomenon of gap closure starting from the bottom (where $\sigma_{N}$ reached its maximum value) with the formation of new GB sections as the grain junction shifts up.
Two complementary mechanisms of accommodation and/or stress relaxation contributing to the gap closure are available:

$$
\sigma_{N}=\frac{M}{\lambda}\left[\frac{\Delta}{2}-\Omega \frac{\Delta}{\omega} \int_{0}^{t_{a}} J_{s}^{\Delta} \partial t\right]=\frac{M}{\lambda}\left(\frac{\Delta_{r}}{2}\right),
$$

which are: [first term in Eq. (1), elastic contribution] grain zipping by inducing a longitudinal strain $\Delta / \lambda=\sigma_{N} / M$ within the grain bulk ( $\Delta / 2$ per grain) with an elastic energy cost $\propto M \omega \Delta^{2}$ [where $M=E /(1-v)$ is the biaxial elastic modulus for isotropic mediums, $E$ is the Young modulus and $v$ the Poisson ratio $^{25}$ ]; and/or (second term, plastic contribution) gap filling by surface downhill currents $J_{s}^{\Delta}=-\left(D_{s} / \Omega k_{B} T\right) \vec{\nabla}_{S} \mu$ biased by the stress-modified surface chemical potential $\mu=$ $-\Omega\left(\kappa \gamma_{s}+\sigma_{N}\right)$ (Ref. 26). Here, $J_{s}^{\Delta}$ flows in the direction in which the density of the strained medium decreases, i.e. from zones under compression $\rightarrow$ unstressed zones (e.g. grain tops) $\rightarrow$ zones under traction. Here, $\Omega$ is the atomic volume, ${ }^{25} D_{s}$ the collective surface diffusion coefficient defined according to the Fick's laws, and $\kappa$ denotes the local curvature of the film surface. For films with high mobility (such that $\lambda^{2} / D_{s} \ll$ $t_{a}$ ), the relaxed fraction of $\sigma_{N}$ is proportional to the product between the transported mass by $J_{s}^{\Delta}$ expressed in number of atoms $\left[(\pi \lambda \Delta / 6) \int_{0}^{t_{a}} J_{s}^{\Delta} \partial t\right.$ where $\pi \lambda \Delta / 6$ is the top aperture of the gap region] and the equivalent gap narrowing per transported atom $\delta \Delta \approx 6 \Omega / \pi \lambda \omega$. In terms of GB motion, a gap narrowing of $\delta \Delta$ is equivalent to a shift up of the grain junction by $\hat{m} \delta \Delta \approx 12 \Omega / \pi \lambda \Delta$. The right-hand expression in Eq. (1) indicates that the grain zipping accommodates only the residual part of the stress $M \Delta_{r} / 2 \lambda$ that is not relaxed by $J_{s}^{\Delta}$, with an elastic energy cost of $M \omega \Delta_{r}^{2}=(\pi \lambda \omega / 6) \gamma_{e}^{\Delta}$, where $\gamma_{e}^{\Delta}$ denotes the elastic energy per gap cross-section unit ascribed to the longitudinal strain.

\section{Azimuthal interactions [Fig. 4(c)]}

Besides the longitudinal interactions through the gap between neighboring grains, interactions in the azimuthal direction have to be considered in the light of the evidence in Fig. 2(c) (that point out to the development of a threefold axis in-plane texture) suggesting the reorientation of the grains during the coalescence. Consequently, the scenario of interactions presented above is completed as follows: as the gap closure proceeds (at a rate $\Omega J_{s}^{\Delta} \sim D_{s} / \Delta$ ) through the mechanisms of gap filling and grain zipping [in Eq. (1)], a torque arises on the buried sections of the opposite faces of the neighboring grains (just below the grain junction) due to the angular misfit (described in terms of the misorientation angle $\theta$ ) between their in-plane orientations. Such a torque promotes the reorientation of the grains via the generation of a shear stress $\sigma_{s} \propto-\nabla_{\theta}\left(\gamma_{g b} / \lambda\right) \cdot \hat{T}$ (with $\nabla_{\theta}$ the surface gradient vector in the azimuthal direction) in order to reduce the GB energy $\gamma_{g b}(\theta)$ through the formation of pseudocoherent GBs. Thus-generated $\sigma_{s}$ is then accommodated/relaxed by means of two complementary mechanisms:

$$
\sigma_{s}=G\left(\frac{\theta}{2}-\Omega \frac{36 \Delta}{\pi^{2} \lambda^{2}} \int_{\omega \Delta / D_{s}}^{t_{a}} J_{s}^{\theta} \partial t\right)=G\left(\frac{\theta_{r}}{2}\right),
$$

which are: [first term in Eq. (2), elastic contribution] by inducing a shear strain of the kind of torsion within the grain bulk with a twist angle per grain of $\theta / 2$ and an energy 
cost $\approx G \omega(\lambda \theta / 2)^{2}$ (where $G$ is the shear modulus ${ }^{25}$ ); and/or [second term in Eq. (2), plastic contribution] by modifying the surface chemical potential as $\mu=-\Omega\left[\kappa \gamma_{s}+\sigma_{N}-\right.$ $\left.\rho\left(S^{\prime}\right) \gamma_{g b}(\theta)\right]$ in the azimuthal direction. Here, $\rho\left(S^{\prime}\right)$ denotes the density of adsorption sites along the grain perimeter $S^{\prime}=\lambda \theta / 2$, whose form changes as the gap closes such that, for a mean-field approach, a time-averaged $\left\langle\rho\left(S^{\prime}\right)\right\rangle=$ $\pi \lambda \omega / 6 \Omega$ is used. From thus-modified $\mu$, a lateral component $J_{s}^{\theta}=-\left(D_{s} / \Omega k_{B} T\right) \vec{\nabla}_{S^{\prime}} \mu=-\left(\pi D_{s} \omega / 3 \Omega k_{B} T\right) \nabla_{\theta} \gamma_{g b} \hat{T}$ of the surface current, which induces a grain rotation, ${ }^{21,22}$ is generated.

Equation (2) puts together information provided by previous studies ${ }^{27-29}$ in agreement with molecular dynamic and phase field simulations ${ }^{21,22,30}$ that demonstrates the key role played by the grain reorientation in processes of coalescence and normal grain growth for polycrystalline systems and in particular for $\mathrm{Au}$ films. ${ }^{21,29}$ In this context, the model assumes elastic deformations of pure torsion (involving shear strain gradients along the grain thickness; see a description of basic kinematics of torsion in Ref. 31) on the basis of considering a $\lambda_{\perp}$-thick shallow layer of surface grains (as discussed above) with circular cross-sections, which are: (i) azimuthally coupled to each other [according to data in Fig. 2(c)] and (ii) clamped at the bottom by the randomly in-plane textured bulk of the polycrystalline film. On the other hand, the fact of considering a lateral component $\vec{J}_{S}^{\theta}$ implies that the net surface current $\vec{J}_{s}=\vec{J}_{s}^{\Delta}+\vec{J}_{s}^{\theta}$ flows (for the case of tensile stress $\sigma_{N}>0$ ) from unstressed grain tops toward the GBs (direction of $\vec{J}_{S}^{\Delta}$ ) along curved trajectories to the right or left in dependence on the $\vec{J}_{s}^{\theta}$ direction [as sketched in Fig. 4(a)]. These trajectories would give rise to an inhomogeneous filling of the gap, which produces a nonuniform advance of the grain faces whose in-plane orientations change consequently. Since the $\vec{J}_{S}^{\theta}$-induced grain rotation involves surface currents rather than GB self-diffusion and/or bulk diffusion phenomena (with $D_{s} \sim 10^{-2}>D_{g b} \sim 10^{-14}>$ $D_{\text {bulk }} \sim 10^{-25} \mathrm{~cm}^{2} / \mathrm{s}$ at $100{ }^{\circ} \mathrm{C}$, respectively $\left.{ }^{32,33}\right)$, this process happens faster (with rates $\leqslant 15^{\circ} / \mathrm{h}$ ) than that expected. ${ }^{34}$ The $J_{s}^{\theta}$-relaxed fraction of $\sigma_{s}$ [second term in Eq. (2)] is estimated as the product between the mass transported by $J_{s}^{\theta}$ expressed in number of atoms $\left[\frac{1}{2} \omega \Delta \int_{\omega \Delta / D_{s}}^{t_{a}} J_{s}^{\theta} \partial t\right.$, where $\omega \Delta$ is the lateral aperture of the gap region] and the rotated angle per transported atom $\left(\approx 72 \Omega / \pi^{2} \lambda^{2} \omega\right.$, details in Ref. 21$)$. The $\lambda$-dependence of the relaxed fraction defines a critical size $\lambda_{0}=\frac{6}{\pi} \sqrt{2 \Omega \frac{\Delta}{\theta} \int_{\omega \Delta / D_{s}}^{t_{a}} J_{s}^{\theta} \partial t}$ for the grain rotation that corresponds to the size of the largest grain in which $J_{s}^{\theta}$ is high enough to fully relax $\sigma_{s}$ during the annealing time, and so $\sigma_{s} \equiv 0$ for subcritical grains (those with sizes $\lambda<\lambda_{0}$ ). Note that there is not analogous critical grain size for the full relaxation of $\sigma_{N}$ through $J_{s}^{\Delta}$. For supercritical grains (with $\lambda>\lambda_{0}$ ), the ability to rotate decreases as $\propto 1 / \lambda^{2}$, in agreement with the simulations in Ref. 22 and so the nonrelaxed fraction of $\sigma_{s}$ [i.e. $G \theta_{r} / 2$ with $\theta_{r}$ being the residual misorientation angle; right-hand term in Eq. (2)] is accommodated elastically by torsion with an energy cost of $G \omega\left(\lambda \theta_{r} / 2\right)^{2}=(\pi \lambda \omega / 6) \gamma_{e}^{\theta}$, where $\gamma_{e}^{\theta}$ denotes the elastic energy per gap cross-section unit ascribed to the shear strain.

\section{Interaction balance}

Once the different contributions to the elastic energy $\left(\gamma_{e}=\gamma_{e}^{\Delta}+\gamma_{e}^{\theta}\right)$ are defined, the balance of the surface-tension forces involved in the interactions between neighboring grains can be described along the out-of-film plane direction as:

$$
\gamma=2 \gamma_{s} \sin [\arctan (\hat{m})]-\gamma_{g b}(\theta)-\gamma_{e}
$$

with $\gamma_{e}^{\Delta}=6 M \Delta_{r}^{2} / \pi \lambda, \quad \gamma_{e}^{\theta}=0$ for $\lambda \leqslant \lambda_{0}$, and $\gamma_{e}^{\theta}=$ $3 G \lambda \theta_{r}^{2} / 2 \pi$ otherwise; where the $\lambda$-dependences of $\Delta_{r}$ and $\theta_{r}$ are given through Eqs. (1) and (2), respectively. Such a balance defines: (a) the resulting force between interacting grains $\gamma \neq$ 0 (where $\gamma>0$ means attraction, repulsion otherwise); and consequently (b) the condition of mechanical equilibrium $\gamma=$ 0 that determines the steady profile $\partial_{t} S(\vec{r})=0$ of the gap region for a selected value $\hat{m}_{0}$ of the border slope such that $2 \gamma_{s} \sin \left[\arctan \left(\hat{m}_{0}\right)\right]=\gamma_{g b}+\gamma_{e}$.

The attraction $(\gamma>0)$ occurs when the surface energy $\left\{2 \gamma_{s} \sin [\arctan (\hat{m})]\right\}$ of the opposed $\hat{m}$-sloped faces of two neighboring grains is higher than the energy involved in the formation of an in-between GB $\left[\gamma_{g b}(\theta)+\gamma_{e}\right]$. Otherwise, the repulsion $(\gamma<0)$ between grains prevails. The gap shape evolves under these interactions until the equilibrium profile (with slope $\hat{m}=\hat{m}_{0}$ ) is reached. Depending on the $\hat{m}_{0}$ value, two limit situations can be outlined: (i) a complete gap closure (denominated also grain coalescence) producing a negligible border slope $\hat{m}_{0} \rightarrow 0$; and (ii) a noncontact for small grains (or grooving regime) for larger ones when $\gamma_{g b}(\theta)+$ $\gamma_{e}>2 \gamma_{s}$, which implies an unreachable equilibrium profile $\left(\hat{m}_{0} \rightarrow \infty\right)$, and so the grains are separated from each other, leaving uncovered regions of the film bulk. For practical purposes, the grain coalescence is supposed when we are not able to distinguish between joined grains (by drawing a well-resolved complete GB). This is equivalent to having $\hat{m}_{0}<\omega_{s} / 5 \delta r$ (the discernible minimum slope) with $\omega_{s}$ and $5 \delta r$ denoting the intrastructure roughness and lateral resolution of the AFM measurements at GB (note that five $\delta r$-sized pixels are required at least to resolve the profile of a gap region), respectively. On the other hand, the appraisable maximum slope is limited by the AFM tip aspect ratio $\left(\approx 2 m_{\text {tip }}\right)$, such that the noncontact/grooving condition can be assumed for $\hat{m}_{0} \approx$ $m_{\text {tip. }}$. At this point, it should be stressed that the balance in Eq. (3) is only suitable for $\hat{m}$ ranged between $\omega_{s} / 5 \delta r$ and $m_{\text {tip. }}$.

Finally, the model assumes that the contributions to the stress relaxation of other mechanisms involving bulk plastic deformations (e.g. motion and generation of dislocations from a Frank-Read source in ductile metals, such as $\mathrm{Au}$ ) play a minor role (and thus negligible). This assessment is valid for both $\sigma_{N}$ and $\sigma_{s}$ being lower than the Au tensile and shear strengths, which were estimated for polycrystalline Au to be $\approx 250 \mathrm{MPa}^{35}$ and $>5 \mathrm{GPa},{ }^{36}$ respectively.

\section{B. Interpretation of our results in light of the model}

\section{Morphology evolution}

The size dependence of the elastic energy $\gamma_{e}$ accumulated as a consequence of the interactions between the surface features is plotted in Fig. 5(a). The solid curve $\gamma_{e}(\lambda)$ corresponds to the best fit of the experimental data (symbols) carried out under the following criteria: (i) according to the model, the 


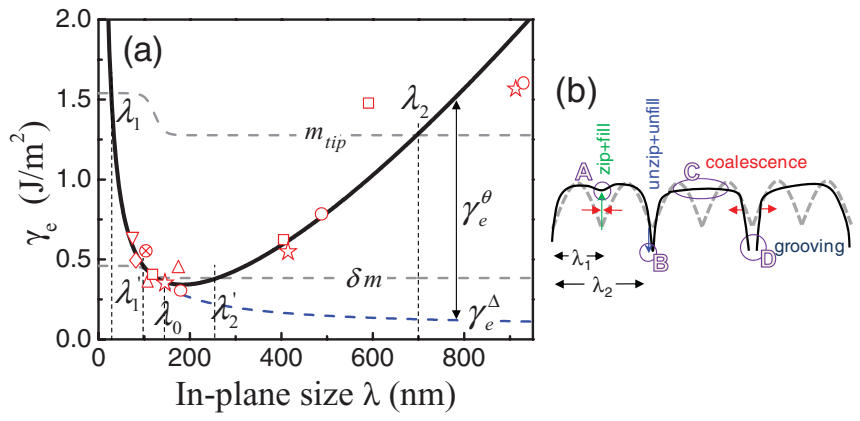

FIG. 5. (Color online) (a) $\lambda$ dependence of $\gamma_{e}$. The solid curve represents the best fit of the experimental results to the model. The symbols correspond to the data measured at different length scales in $200 \mathrm{~nm}$-thick polycrystalline $\mathrm{Au}$ films with annealing times of $(\nabla) t_{a}=$ $0 \mathrm{~s}$ (as-grown), $(\otimes) t_{a}=1.2 \times 10^{2} \mathrm{~s},(\diamond) 3 \times 10^{2} \mathrm{~s},(\square) 3 \times 10^{3}$ $\mathrm{s},(\star) 1.2 \times 10^{4} \mathrm{~s}$, and (०) $1.2 \times 10^{5} \mathrm{~s}$. (b) Sketch of the evolution of a surface profile (the dashed and solid curves correspond to preand post-coalescence profiles, respectively) in which the morphology transformations that prevail at different-length scales are indicated (see description in the text).

surface of the as-grown Au film is formed by small grains with sizes $\lambda_{\text {as-grown }} \ll \lambda_{0}$, which can rotate freely $\left(\gamma_{g b} \approx 0\right)$ such that, from the equilibrium condition, we get $\gamma_{e}\left(\lambda_{\text {as }- \text { grown }}\right)=$ $2 \gamma_{s} \sin \left[\arctan \left(\hat{m}_{\text {as-grown }}\right)\right]$ with $\lambda_{\text {as-grown }}$ and $\hat{m}_{\text {as-grown }}$ measured by AFM [Figs. 1(f) and 1 (g)] and $\gamma_{s}$ computed by EAM. ${ }^{23}$ This assumption is kept for all the subcritical grains (those with $\lambda \leqslant$ $\lambda_{0}$ that do not accumulate shear strain, $\gamma_{e}^{\theta} \approx 0$ ). (ii) $\lambda_{0}$ is taken as the largest length scale [see Fig. 2(d)] in which the threefold axis in-plane texture persists along the annealing. (iii) The equilibrium condition for supercritical grains becomes $\gamma_{e}(\lambda>$ $\left.\lambda_{0}\right)=2 \gamma_{s} \sin [\arctan (\hat{m})]-\gamma_{g b}^{\mathrm{CSL}}$, where $\gamma_{g b}^{\mathrm{CSL}}=0.304 \mathrm{~J} / \mathrm{m}^{2}$ is the mean GB energy averaged from the relative populations ascribed to each kind of GB [Fig. 3(a)] once their respective energies are considered [namely $\gamma_{g b}\left(\theta=0^{\circ}\right), \gamma_{g b}\left(7^{\circ}\right)$, and $\gamma_{g b}\left(13^{\circ}\right)$; Fig. $\left.3(\mathrm{~b})\right]$ computed by EAM..$^{23}$ The $\lambda$ dependences of $\Delta_{r}$ and $\theta_{r}$ (the fitting parameters) are calculated from considerations (i) and (iii). As expected, such dependences fulfil the asymptotic conditions $\Delta_{r}(\lambda \rightarrow 0) \rightarrow 0\left[\theta_{r}\left(\lambda \leqslant \lambda_{0}\right)=0\right]$ and $\Delta_{r}(\lambda \rightarrow \infty) \rightarrow \Delta\left[\theta_{r}(\lambda \rightarrow \infty)=\theta\right]$ that point out to the fact that the longitudinal [shear] stress is relaxed or accommodated mainly via surface currents or strain generation in small $(\lambda \rightarrow 0)$ and large $(\lambda \rightarrow \infty)$ grains, respectively. The asymptotic condition $\lambda \rightarrow \infty$ allows us to estimate the mean values of the gap width $\langle\Delta\rangle$ and the misorientation angle $\langle\theta\rangle$ between surface features. The dashed horizontal lines in Fig. 5(a) correspond to the coalescence (labeled $\delta m)$ and noncontact/grooving $\left(m_{\text {tip }}\right)$ conditions, as defined in the previous section. Their intercepts with the curve $\gamma_{e}(\lambda)$ [explicitly, $\lambda_{1}\left(\lambda_{1}^{\prime}\right)$ and $\lambda_{2}\left(\lambda_{2}^{\prime}\right)$ for the line $m_{\text {tip }}(\delta m)$ ] define the interaction regimes between the surface features as a function of their sizes. The morphological parameters $\langle\Delta\rangle,\langle\theta\rangle$, and the $\lambda$ intercepts obtained from the fit are summarized in Table I.

Figure 5(a) allows us to interpret the experimental data in Figs. 1-3 in light of the proposed $\operatorname{model}^{37}$ as follows [the description is assisted by the sketch in Fig. 5(b)].

(i) For grains smaller than the critical size for the formation of grain boundaries $\lambda_{g} \leqslant \lambda_{1}$ \{such that $\gamma_{e}\left(\lambda_{g}\right) \geqslant 2 \gamma_{s}$
TABLE I. Summary of the morphology parameters obtained from the numerical fit of the experimental data to model (except $\lambda_{0}$ that corresponds to an input parameter). The physical and geometric constants used to compute the model are summarized in Ref. 25.

\begin{tabular}{lc}
\hline Morphological parameters & \\
\hline$\lambda_{0}$ & $136.0 \mathrm{~nm}$ \\
$\lambda_{1}$ & $27.7 \mathrm{~nm}$ \\
$\lambda^{\prime}{ }_{1}$ & $98.0 \mathrm{~nm}$ \\
$\lambda^{\prime}{ }_{2}$ & $256.1 \mathrm{~nm}$ \\
$\lambda_{2}$ & $697.5 \mathrm{~nm}$ \\
$\langle\Delta\rangle$ & $0.45 \mathrm{~nm}$ \\
$\langle\theta\rangle$ & $3.2^{\circ}$ \\
\hline \hline
\end{tabular}

$\sin \left[\arctan \left(m_{\text {tip }}\right)\right]$, which means an unreachable equilibrium condition $\}$, the gap between grains is too wide $(\Delta \sim \lambda)$ to be filled and/or zipped. The grains do not interact with each other (noncontact regime), and then a compressive growth stress arises from the surface tension on isolated grains. Here, $\lambda_{1}=27.7 \mathrm{~nm}$ (in Table I), estimated from the fit, agrees with the sizes (ranged between $20-30 \mathrm{~nm}$ ) of the isolated grains (before these come into contact with each other) measured in $\approx 20$-nm-thick films grown according to $\mathrm{VW}$ mode. ${ }^{10,14,38}$ This regime is outside the scope of our study as the grain size for all our samples is $\lambda_{g}>\lambda_{1}$.

(ii) For grains with sizes $\lambda_{1}<\lambda_{g}<\lambda_{0}$ \{such that $\gamma_{e}\left(\lambda_{g}\right)<2 \gamma_{s} \sin \left[\arctan \left(m_{\text {tip }}\right)\right]$, available equilibrium $\}$, the pre-equilibrium $\gamma>0$ as $\gamma_{e}$ drops with $\lambda_{g}$, according to Eq. (3). This means the attraction between the opposed faces of neighboring grains predominates giving rise to a progressive gap closure [transformation A in Fig. 5(b)] by gap filling and grain zipping plus free rotation in the case of in-plane misorientation. Indeed, the existence of downhill currents $J_{s}^{\Delta}$ flowing toward the GBs (gap filling) and/or the fact that grains are under residual in-plane traction (grain zipping) are consistent with the morphology evolution obtained for the early regime [Figs. 1(c) and 2(c)]. The gap-closure rate with $\lambda$ estimated from Eq. (3) becomes $\partial \hat{m}_{0} / \partial \lambda \approx\left(1 / 2 \gamma_{s}\right) \partial \gamma_{e} / \partial \lambda$ for $\hat{m}_{0} \ll 1$, which means that $\hat{m}_{0}$ exhibits a similar behavior to that of $\gamma_{e}$ vs $\lambda$, i.e. $\hat{m}_{0}$ drops as $\hat{m}_{0} \propto A / \lambda$ (with $A=$ $3 M\langle\Delta\rangle^{2} / \pi \gamma_{s}$ ) down to $A / \lambda_{0}$.

Consequently, when $A / \lambda_{0}<\hat{m}_{0} \leqslant \delta m$, which is fulfilled for $\lambda^{\prime}{ }_{1} \leqslant \lambda_{g}<\lambda_{0}$ with $\gamma_{e}\left(\lambda_{g}\right) \leqslant 2 \gamma_{s} \sin [\arctan (\delta m)]$, the coalescence of the grains that interact through the gap is supposed [transformation $\mathrm{C}$ in Fig. 5(b)]. As a result of such coalescence, the surface structures with sizes $\lambda_{s}=$ $n \lambda_{g}>\lambda_{g}$ are formed, and the gaps that are still visible on the structures between the coalesced grains become internal GBs. The fact that the highest normal stress generated during the coalescence $\sigma_{N}\left\langle M\langle\Delta\rangle / \lambda_{0} \approx 225 \mathrm{MPa}\right.$, with $\langle\Delta\rangle \approx$ $0.45 \mathrm{~nm}$ computed from the asymptotic conditions once the data is fitted (Table I), is lower than the Au tensile strength $\left(\approx 250 \mathrm{MPa}^{35}\right)$ confirms the model's assumption concerning the minor role played by the bulk plastic deformations on the stress relaxation. Note that the threshold border slope $(\delta m \approx$ 0.15 ) used to define the coalescence condition was fixed to a slightly higher value than that corresponding to the discernible minimum slope $\left(\omega_{s} / 5 \delta r=1.25 \mathrm{~nm} / 10 \mathrm{~nm}=0.125\right)$ described 
in the previous section, which allows us to investigate the morphology evolution of the inner grains.

The main repercussion of the formation of the surface structures is the fact that two different-length scales of interaction appear: whereas the intrastructure interactions are controlled by the single properties of the inner $\lambda_{g}$-sized grains, the structures interact with each other as a homogeneous whole of size $\lambda_{s}$ exhibiting the mean properties of the set of grains forming them. This difference resides in the better mechanical coupling between inner grains through the shallow internal GBs than that taking place between the structures through deeper external GBs. The experimental data measured at different-length scales for a single sample [as shown in Fig. 1(e)-1(g)] are plotted in Fig. 5(a) as different points all displayed using the same symbol (i.e. one symbol per sample).

(iii) For surface features (grains and structures) with sizes $\lambda_{0}<\lambda<\lambda_{2}$, the increase in $\gamma_{e}$ accounts for the progressive loss of the ability to relax fully the in-plane misorientation via $J_{s}^{\theta}$-induced rotation of the coalescing features. The nonrelaxed part is then accommodated by shear strain with the subsequent energy cost. Thus, as the elastic energy is accumulated, pre-equilibrium $\gamma<0$, which implies that the repulsion between the opposed faces of the close features prevails. Such repulsion would induce a shrinking of the interacting features, giving rise to deeper and sharper gaps between them (transformation B) and the worsening of the threefold axis in-plane texture due to the shear-strain release. Note that this description is in good agreement with the morphology evolution obtained during the late regime [Figs. 1(d) and 2(d)] for the surface structures. The shrinkage of the structures results foreseeable from partial unzipping processes (major effect) and uphill currents $J_{s}^{\Delta}$ (minor one) emptying the external GBs. The shrinking rate $\left\{\partial \hat{m}_{0} / \partial \lambda=\left[\left(1+\hat{m}_{0}^{2}\right)^{3 / 2} / 2 \gamma_{s}\right] \partial \gamma_{e} / \partial \lambda \approx\right.$ $B\left(1+\hat{m}_{0}^{2}\right)^{3 / 2}$ with $B=3 G\langle\theta\rangle^{2} / 4 \pi \gamma_{s}$ from Eq. (3) $\}$ increases progressively with $\lambda$ from $\partial \hat{m}_{0} / \partial \lambda \approx\left(1+\delta m^{2}\right)^{3 / 2} B \approx B$ for $\lambda_{0}<\lambda<\lambda_{2}^{\prime}$ (assuming $\left.\delta m \ll 1\right)$ up to $\partial \hat{m}_{0} / \partial \lambda \approx(1+$ $\left.m_{\text {tip }}^{2}\right)^{3 / 2} B \approx 1.7 B$ for $\lambda \rightarrow \lambda_{2}$. The fact that the highest shear stress $\sigma_{s}=G\langle\theta\rangle / 2 \approx 0.8 \mathrm{GPa}$ generated by the mean misorientation between neighboring features, which was estimated to be $\langle\theta\rangle=3.2^{\circ}$ (Table I), is lower than the shear strength reported for polycrystalline $\mathrm{Au}$ films $\left(>5 \mathrm{GPa}^{36}\right.$ ) supports the model soundness.

(iv) When $\hat{m}_{0}$ reaches a value similar to $m_{\text {tip }}$, which happens for surface features with sizes higher than the critical size for the formation of surface discontinuities, $\lambda \geqslant \lambda_{2}$ \{such that $\gamma_{e}\left(\lambda_{g}>\lambda_{2}\right) \geqslant 2 \gamma_{s} \sin \left[\arctan \left(m_{\text {tip }}\right)\right]$, unreachable equilibrium $\}$, the structures become fully separated from each other, and the in-between GBs disappear, leaving uncovered regions of the film bulk [surface discontinuities; transformation D in Fig. 5(b)]. Since the shrinking of the isolated structures would counterbalance their lateral growths to avoid these coming into contact, $\lambda_{2}$ can be realized as the saturation size of such structures. Phenomena of the saturation of the feature size in bulk as well as on the surface have been reported for polycrystalline films during growth and annealing. ${ }^{38}$ The value estimated here of $\lambda_{2}=697.5 \mathrm{~nm}$ (Table I) is in good agreement with that $\left(\approx 650 \mathrm{~nm}^{38}\right)$ found in $\mathrm{Au} / \mathrm{SiO}_{2}$ films with longer annealing $\left(t_{a} \approx 1.8 \times 10^{4} \mathrm{~s}\right)$ once $50 \%$ of the surface grains had coalesced into the multigrain structures. Nevertheless, since the high-crystalline coherence of the largest multigrain structures (with low-angle and CSL internal GBs), that would be translated in a decrease of both the tensile and shear strengths, the presence of plastic deformations generated by dislocation motion within the structure bulk cannot be neglected. As a consequence of these plastic deformations, the growth stress would be relaxed complementarily, the accumulated elastic energy would diminish, and saturation sizes $\lambda_{2}$ larger than that estimated here could be expected.

Beyond the local mechanical equilibrium [in Eq. (3)] established between neighboring grains through a GB emerging at an $\hat{m}_{0}$-sloped gap region, the shrinkage of the surface structures can be understood as the result of the attraction (which is unbalanced along the structure radius) between the inner grains. Thus, for a given grain, the attraction that it experiences for the neighboring grain closer to the structure center is higher than the interaction with the more distant one. This asymmetry is a consequence of the accumulation of elastic energy $\gamma_{e}$ with $\lambda$ [Fig. 5(a) for $\lambda>\lambda_{0}$ ], where $\lambda$ can be realized here as the interaction length rather than the grain size. The attraction is thus weakened as the distance from the structure center increases until repulsion due to the prevalence of the opposite attraction toward the centers of the neighboring structures. Therefore, the $\lambda$ dependence of the interactions between the surface features acts like an inside grain-bundling/outside grooving mechanism. The fact that the stress induced by the intrastructure attraction between the inner grains is mostly relaxed by means of surface currents (plastic effect), whereas that generated by interstructure repulsion is elastically accommodated via generation of strain-that is, the physical parameter investigated to determine the residual intrinsic stress within the samples-would address the compressive nature of the postcoalescence growth stress. The dependence of such a stress on the flux during growth ${ }^{7,8}$ (that it is not our case) would be given as a result of transient changes induced by the flux in the equilibrium steady profiles of the gap regions, changes that are relaxed by the surface currents once the flux is stopped.

\section{Grain growth kinetics}

Usually, the grain growth kinetics are described in terms of GB migration driven by the minimization of the system energy via: ${ }^{12}$ (for bulk grains) the reduction of the total amount of GB energy, which implies reducing the total area of boundary and/or the formation of low-angle and CSL GBs; (for surface grains) modifying the balance between GB energy and free-surface energy through processes of gap closure and grooving involving in-plane reorientations. In this context, it is assumed that the grains grow at a rate $\partial \lambda_{g} / \partial t \approx$ $M_{g b} \Theta$, where $M_{g b}$ and $\Theta$ denote the GB mobility and the driving force for the GB migration, respectively. For bulk (surface) grains it holds that $\Theta \propto \kappa\left(\propto \kappa_{s}\right)$ is proportional to the grain curvature $\kappa \approx \lambda_{g}^{-1}$ (the grain curvature projected on the film surface $\kappa_{s} \approx \nabla_{s}^{2} \lambda_{g}^{-1}$ ), such that for an ideal growth $M_{g b}$ is independent of $\lambda_{g}$ and so $\lambda_{g} \propto t^{p}$ with a coarsening exponent $p=1 / 2(p=1 / 4)$. For real growth, lower $p$ are expected since $M_{g b}$ decreases as a consequence of boundary pinning processes and/or boundary migration involving complex movements. ${ }^{39}$ The coarsening exponents obtained in our films $\left(\lambda_{g} \propto t^{0.14 \pm 0.02}\right.$ for the in-plane surface 
grain size and $\lambda_{\perp} \propto t^{0.10 \pm 0.01}$ for the out-of-plane size of coherent surface features, in previous section) are much lower than $1 / 4$, which indicates (in agreement with our experimental evidences) that grain growth is ruled by the surface diffusion and thus limited to a shallow layer of grains. In addition to boundary migration along the out-of-plane direction that is inherent in the gap closure that gives rise to the coalescence, a nonuniform closure [like that generated by the reorientation of the coalescing grains; sketch in Fig. 4(b)] generates also an in-plane boundary migration at a rate comparable to the $J_{s}^{\theta}$ induced grain rotation rate. Such migration interferes (delays) in the in-plane boundary migration responsible for surface grain growth. Based on these considerations, the GB mobility can be estimated according to Eq. (2) (second bracketed term) to be $M_{g b} \sim \partial \theta / \partial t=36 \Omega \Delta J_{s}^{\theta} / \pi^{2} \lambda_{g}^{2}$. Assuming then that $J_{s}^{\theta}$ decays as $1 / \lambda_{g}^{\beta}$ with $\beta=1$ (Refs. 12 and 13) for surface currents on grains larger than the diffusion length or $\beta=2$ for perimeter currents along the grain boundary, ${ }^{21}$ we get $M_{g b} \propto$ $1 / \lambda_{g}^{2+\beta} \Rightarrow \lambda_{g} \propto t^{p}$ with $p \approx 1 / 7-1 / 8$ for GB migration, giving rise simultaneously to both surface grain growth and in-plane grain reorientation. Note that the thus-estimated range for the coarsening exponent is in excellent agreement with our results.

Investigations concerning the kinetics of formation of the large surface structures from random gap-closure events as well as the determination of the configurations of mechanical equilibrium for polycrystalline thin film bonds to stiffener substrates (such that the films cannot be considered as free standing) by finite element methods are in progress.

\section{CONCLUSIONS}

Here, we report experimental findings concerning the local interactions between grains that coarsen on the surface of annealed polycrystalline $\mathrm{Au}(111)$ films. In light of these findings, we have proposed a comprehensive model that addresses successfully the morphological transformations observed in the annealed films and offers a plausible explanation for the compressive nature of the growth stress generated during the postcoalescence. Such an explanation is based on the following evidence and/or arguments: The findings point to the fact that the grain coarsening occurs mostly by coalescence of singlecrystal grains into multigrain structures (rather than by grain growth) involving progressively mechanisms of relaxation by surface currents and accommodation by strain of the growth stress generated in both the normal and azimuthal directions. As the surface features (i.e. grains and structures) become larger, the ratio of accommodated stress to relaxed stress rises with the resulting increment in the elastic energy (in particular that ascribed to the shear strain generated during the coalescence of larger misoriented structures). Consequently, the balance between the energy saved by the system as a result of the coalescence and the accumulated elastic energy at differentlength scales determines the nature of the local interactions. Thus, the fact that such a balance is favorable to the coalescence at grain-size scale explains the tendency to bundle grains into structures; whereas the unfavorable balance at structuresize scale accounts for the repulsion between the structures, this being the origin of the compressive growth stress.

\section{ACKNOWLEDGMENTS}

This paper was supported by the projects F1-54173 (bilateral program CSIC-Conacyt) 200960I182 (CSIC), and CCG10-UAM/MAT-5537 (DGUI-Comunidad de Madrid and Universidad Autónoma de Madrid). A.G.G. acknowledges the financial support of the MICINN Spanish Ministry under the project ESP2006-14282-C02-02. *agonzo@icmm.csic.es

${ }^{1}$ J. A. Floro, S. J. Hearne, J. A. Hunter, P. Kotula, E. Chason, S. C. Seel, and C. V. Thompson, J. Appl. Phys. 89, 4886 (2001).

${ }^{2}$ L. B. Freund and S. Suresh, Thin Film Materials: Stress, Defect Formation and Surface Evolution (Cambridge University Press, Cambridge, England, 2003), pp. 44-166.

${ }^{3}$ R. C. Cammarata, T. M. Trimble, and D. J. Srolovitz, J. Mater. Res. 15, 2468 (2000).

${ }^{4}$ W. D. Nix and B. M. Clemens, J. Mater. Res. 14, 3467 (1999).

${ }^{5}$ R. Koch, D. Hu, and A. K. Das, Phys. Rev. Lett. 94, 146101 (2005).

${ }^{6}$ J. Leib and C. V. Thompson, Phys. Rev. B 82, 121404 (2010).

${ }^{7}$ R. Koch, J. Phys. Condens. Matter 6, 9519 (1994).

${ }^{8}$ E. Chason, B. W. Sheldon, and L. B. Freund, Phys. Rev. Lett. 88, 156103 (2002).

${ }^{9}$ C. Friesen and C. V. Thompson, Phys. Rev. Lett. 89, 126103 (2002).

${ }^{10}$ C. Polop, C. Rosiepen, S. Bleikamp, R. Drese, J. Mayer, A. Dimyati, and T. Michely, New J. Phys. 9, 74 (2007).

${ }^{11}$ S. Ruan and C. A. Schuh, J. Appl. Phys. 107, 073512 (2010) and references therein.

${ }^{12}$ M. Z. Allmang, L. C. Feldman, and M. H. Grabow, Surf. Sci. Rep. 16, 377 (1992).
${ }^{13}$ A. L. Barabási and H. E. Stanley, Fractal Concepts in Surface Growth (Cambridge University Press, Cambridge, England, 1995), pp. 139-145.

${ }^{14}$ M. J. Rost, D. A. Quist, and J. W. M. Frenken, Phys. Rev. Lett. 91, 026101 (2003)

${ }^{15}$ J. A. Thornton, J. Vac. Sci. Technol. 11, 666 (1974).

${ }^{16}$ Z. Zhong, W. Schwinger, F. Schäffler, G. Bauer, G. Vastola, F. Montalenti, and L. Miglio, Phys. Rev. Lett. 98, 176102 (2007).

${ }^{17}$ B. Sanduijav, D. Matei, G. Chen, and G. Springholz, Phys. Rev. B 80, 125329 (2009) and references therein.

${ }^{18}$ E. Palacios-Lidón, L. Guanter, J. Zúñiga-Pérez, V. Muñoz-Sanjosé, and J. Colchero, Small 3, 474 (2007).

${ }^{19}$ A. González-González, M. Alonso, E. Navarro, J. L. Sacedón, and A. Ruiz, Nanoscale Res. Lett. 5, 1882 (2010).

${ }^{20}$ C. V. Thompson and R. Carel, Mat. Sci. Eng. B 32, 211 (1995).

${ }^{21}$ K. E. Harris, V. V. Singh, and A. H. King, Acta Mater. 46, 2623 (1998).

${ }^{22}$ M. Upmanyu, D. J. Srolovitz, A. E. Lobkovsky, J. A. Warren, and W. C. Carter, Acta Mater. 54, 1707 (2006).

${ }^{23}$ J. Mei, J. W. Davenport, and G. W. Fernando, Phys. Rev. B 43, 4653 (1991).

${ }^{24}$ Y. N. Wen and J. M. Zhang, Solid State Commun. 144, 163 (2007). 
${ }^{25}$ Elastic constants: (Young modulus) $E=79 \mathrm{GPa}$, (Poisson ratio) $v=$ 0.44 , (shear modulus) and $G=27 \mathrm{GPa}$; structural and geometric parameters: (atomic volume) $\Omega=12.5 \times 10^{-3} \mathrm{~nm}^{3}$, (Pixel size) $\delta r=2 \mathrm{~nm}$, [average misorientation between grains on randomly textured $\mathrm{Au}(111)] \bar{\theta}=15^{0}$, (appraisable maximum slope) $m_{\text {tip }} \approx$ $0.6 \pm 0.1$, (threshold border slope) $\delta m \approx 0.15 \pm 0.05$, and (intragrain roughness) $\omega_{g} \approx 1.8 \mathrm{~nm}$. Thermodynamic constants: (surface energy) $\gamma_{s}=1.54 \mathrm{~J} / \mathrm{m}^{2}$, and (mean CSL GB energy for our films) $\gamma_{g b}^{\mathrm{CSL}}=0.34 \mathrm{~J} / \mathrm{m}^{2}$ computed by EAM (Ref. 23) in agreement with data in Ref. 24.

${ }^{26}$ J. S. Tello, A. F. Bower, E. Chason, and B. W. Sheldon, Phys. Rev. Lett. 98, 216104 (2007).

${ }^{27}$ V. Randle, Philos. Mag. A 67, 1301 (1993).

${ }^{28}$ T. Yamasaki, Y. Demizu, and Y. Ogino, Mater. Sci. Forum 204, 461 (1996).

${ }^{29}$ P. Liu, S. C. Mao, L. H. Wang, X. D. Han, and Z. Zhang, Scr. Mater. 64, 343 (2011).

${ }^{30}$ D. Moldovan, D. Wolf, and S. R. Phillpot, Acta Mater. 49, 3521 (2001); D. Moldovan, D. Wolf, S. R. Phillpot, and A. J. Haslam, ibid. 50, 3397 (2002).

${ }^{31} \mathrm{G}$. Timoshenko, Mechanics of Materials (PWS Publications, Boston, 1997), pp. 139-180.

${ }^{32}$ C. L. Liu, Surf. Sci. 253, 334 (1991).

${ }^{33}$ D. Gupta, J. Appl. Phys. 44, 4455 (1973); Phys. Rev. B 7, 586 (1973).
${ }^{34} \mathrm{~A}$ grain rotation rate of $2.4^{\circ} / \mathrm{h}$ involving GB self-diffusions was estimated for $66 \mathrm{~nm}$-sized grains within the bulk of a polycrystalline $\mathrm{Au}(111)$ film (Ref. 21); whereas for high-mobility GBs with enhanced dislocation/disclination activity, a higher rotation rate of $10^{\circ} / \mathrm{h}$ was reported in Ref. 29.

${ }^{35}$ Y. Cao, S. Allameh, D. Nankivil, S. Sethiaraj, T. Otiti, and W. Soboyejo, Mater. Sci. Eng. A 427, 232 (2006).

${ }^{36}$ J. D. Kiely and J. E. Houston, Phys. Rev. B 57, 12588 (1998).

${ }^{37}$ The function to fit the data was built from Eq. (3) as: $\gamma_{e}(\lambda)=$ $\gamma_{e}^{\Delta}(\lambda)+\gamma_{e}^{\theta}(\lambda)=6 M \Delta_{r}^{2}(\lambda) / \pi \lambda+G \lambda \theta_{r}^{2}(\lambda) \cdot \psi\left(\lambda-\lambda_{0}\right) / 2 \pi$ with: (a) $\Delta_{r}(\lambda)=\Delta \cdot \beta^{\prime}(\lambda)$, where the fitting function $\beta^{\prime}(\lambda) \propto 1-$ $2(\Omega / \omega) \int_{0}^{t_{a}} J_{s}^{\Delta} \partial t$ (according Eq. (1)) denotes the non-relaxed fraction of the normal stress by surface currents. The dependence of $\beta^{\prime}$ on $\lambda$ results from the implicit $\lambda$-dependence of $J_{s}^{\Delta}$. Thus-defined $\beta^{\prime}(\lambda)$ fulfils the following condition: $\beta^{\prime}(\lambda) \leqslant 1$ with $\beta^{\prime}(\lambda \rightarrow \infty) \rightarrow$ 1. (b) $\theta_{r}(\lambda)=\theta \cdot \beta^{\prime \prime}(\lambda)$, where the fitting function $\beta^{\prime \prime}(\lambda) \propto 1-$ $72\left(\Omega \Delta / \pi^{2} \theta\right) \int_{\omega \Delta / D_{s}}^{t_{a}}\left(J_{s}^{\theta} / \lambda^{2}\right) \partial t$ (according Eq. (2)) denotes the non-relaxed fraction of the shear stress, its dependence on $\lambda$ being a consequence of the $\lambda$-dependence of $J_{s}^{\Delta} / \lambda^{2}$. Similarly, $\beta^{\prime \prime}(\lambda) \leqslant 1$ with $\beta^{\prime \prime}\left(\lambda=\lambda_{0}\right)=0$ and $\beta^{\prime \prime}(\lambda \rightarrow \infty) \rightarrow 1$. (c) $\psi\left(\lambda-\lambda_{0}\right)$ the step function defined as: $\psi(x)=1$ for $x \geqslant 0$ and $\psi(x)=0$ otherwise. It is assumed that both $\beta^{\prime}(\lambda)$ and $\beta^{\prime \prime}(\lambda)$ depend smoothly on $\lambda$.

${ }^{38}$ C. C. Wong, H. I. Smith, and C. V. Thompson, Appl. Phys. Lett. 48, 335 (1986).

${ }^{39}$ V. Y. Novikov, Scr. Mater. 55, 243 (2006). 